\title{
Reproducibility of the NanoString 22-gene molecular subgroup assay for improved prognostic prediction of medulloblastoma
}

\author{
Letícia F. Leal, ${ }^{1 *}$ Adriane F. Evangelista, ${ }^{1 *}$ Flávia E. de Paula, ${ }^{2 *}$ Gisele Caravina Almeida, ${ }^{3}$ \\ Adriana C. Carloni, ${ }^{1}$ Fabiano Saggioro, ${ }^{4}$ João N. Stavale, ${ }^{5}$ Suzana M.F. Malheiros, ${ }^{5}$ Bruna Mançano, ${ }^{6}$ \\ Marco A. de Oliveira, ${ }^{7}$ Betty Luu, ${ }^{8}$ Luciano Neder, ${ }^{1,3}$ Michael D. Taylor ${ }^{8}$ and Rui M. Reis ${ }^{1,2,9,10}$ (D) \\ ${ }^{1}$ Molecular Oncology Research Center, ${ }^{2}$ Molecular Diagnostic Laboratory, ${ }^{3}$ Department of Pathology, ${ }^{6}$ Children and \\ Young Adult's Cancer Hospital, ${ }^{7}$ Statistics Unity, Barretos Cancer Hospital, Barretos, ${ }^{4}$ Department of Pathology, \\ Ribeirao Preto Medical School, University of São Paulo, Ribeirao Preto, ${ }^{5}$ Department of Pathology, Universidade \\ Federal de São Paulo, São Paulo, Brazil, ${ }^{8}$ Hospital for Sick Children, University of Toronto, Toronto, Ontario, Canada, \\ ${ }^{9}$ Life and Health Sciences Research Institute (ICVS), Health Sciences School, University of Minho, Braga and ${ }^{10}$ ICVS/ \\ 3B's-PT Government Associate Laboratory, Braga/Guimarães, Portugal
}

Medulloblastoma is the most frequent malignant brain tumor in children. Four medulloblastoma molecular subgroups, $\mathrm{MB}_{\mathrm{SHH}}, \mathrm{MB}_{\mathrm{WNT}}, \mathrm{MB}_{\mathrm{GRP} 3}$ and $\mathrm{MB}_{\mathrm{GRP} 4}$, have been identified by integrated high-throughput platforms. Recently, a 22-gene panel NanoString-based assay was developed for medulloblastoma molecular subgrouping, but the robustness of this assay has not been widely evaluated. Mutations in the gene for human telomerase reverse transcriptase (hTERT) have been found in medulloblastomas and are associated with distinct molecular subtypes. This study aimed to implement the 22-gene panel in a Brazilian context, and to associate the molecular profile with patients' clinical-pathological features. Formalin-fixed, paraffin-embedded (FFPE) medulloblastoma samples $(n=104)$ from three Brazilian centers were evaluated. Expression profiling of the 22-gene panel was performed by NanoString and a Canadian series $(n=240)$ was applied for training phase. hTERT mutations were analyzed by PCR followed by direct Sanger sequencing and the molecular profile was associated with patients' clinicopathological features. Overall, $65 \%$ of the patients were male, average age at diagnosis was 18 years and $7 \%$ of the patients presented metastasis at diagnosis. The molecular

Correspondence: Rui M. Reis, PhD, Molecular Oncology Research Center, Barretos Cancer Hospital, Rua Antenor Duarte Villela, 1331; CEP 14784 400, Barretos, S. Paulo, Brazil. Email: ruireis. hcb@gmail.com

*LFL, AFE and FEP equally contributed to this work.

Received 06 April 2018; Revised 22 June 2018; Accepted 25 July 2018; published online 28 August 2018.

(C) 2018 Japanese Society of Neuropathology classification was attained in $100 \%$ of the cases, with the following frequencies: $\mathrm{MB}_{\mathrm{SHH}}(n=51), \mathrm{MB}_{\mathrm{WNT}}(n=19)$, MB $_{\text {GRP4 }}(n=19)$ and $M_{\text {GRP3 }}(n=15)$. The MB $B_{\text {SHH }}$ and MB $_{\text {GRP3 }}$ subgroups were associated with older and younger patients, respectively. The MB GRP4 $_{\text {subgroup }}$ exhibited the lowest 5-year cancer-specific overall survival (OS), yet in the multivariate analysis, only metastasis at diagnosis and surgical resection were associated with OS. hTERT mutations were detected in $29 \%$ of the cases and were associated with older patients, increased hTERT expression and $\mathrm{MB}_{\mathrm{SHH}}$ subgroup. The 22-gene panel provides a reproducible assay for molecular subgrouping of medulloblastoma FFPE samples in a routine setting and is well-suited for future clinical trials.

Key words: $\mathrm{h} T E R T$, medulloblastoma, molecular subgroups, NanoString.

\section{INTRODUCTION}

Medulloblastoma is the most frequent malignant brain tumor in children, accounting for approximately $20 \%$ of all pediatric intracranial tumors. ${ }^{1,2}$ Recent estimates of the occurrence of central nervous system tumors, such as medulloblastoma, suggest they represent about $2.5 \%$ of the newly diagnosed cases of pediatric neoplasms in Brazil. ${ }^{3}$ In adults, medulloblastomas are less common, with a peak of incidence between 20 and 35 years old. ${ }^{4}$ Advances in the treatment of medulloblastoma have improved survival rates over the last few decades. However, the disease outcome remains very poor, with both 5-year overall and 
event-free survivals for high-risk patients being about $25-44 \% .^{5-7}$

Histologically, medulloblastomas can be classified as classic, the most common subtype $(70 \%)$, followed by the desmoplastic (10-20\%), anaplastic/large-cell subtype $(5-10 \%)$ and extensive nodularity subtype (5-10\%). ${ }^{5,8}$ The major prognostic factors are histology, age at diagnosis, presence of metastatic disease, and the extent of tumor volume at resection. ${ }^{9,10}$ Younger medulloblastoma patients, who have evidence of metastasis at diagnosis, residual disease after surgery or large cells/anaplastic histology, are associated with an unfavorable prognosis..$^{9,11,12}$ Until recently, these clinical and pathological prognostic factors were used for defining overall disease risk, but newer combinations of clinical, pathological and molecular prognostic markers are providing a more accurate disease risk stratification. ${ }^{13}$

Medulloblastomas can be molecularly classified based on specific genomic and genetic features. ${ }^{14-16}$ Gene expression analysis using large cohorts of medulloblastomas has identified four molecular subgroups of medulloblastoma, namely WNT ( $\left.\mathrm{MB}_{\mathrm{WNT}}\right)$, SHH $\left(\mathrm{MB}_{\mathrm{SHH}}\right)$, group $3\left(\mathrm{MB}_{\mathrm{GRP} 3}\right)$ and group $4\left(\mathrm{MB}_{\mathrm{GRP}}\right) \cdot{ }^{14,16,17}$ Moreover, this molecular classification correlates with several clinicalpathological features, such as patient prognosis, histology and age at diagnosis. ${ }^{10,17}$ The $\mathrm{MB}_{\mathrm{WNT}}$ subgroup has a better prognosis, whereas $\mathrm{MB}_{\mathrm{GRP} 3}$ patients present with a poor prognosis. ${ }^{18} \mathrm{MB}_{\mathrm{WNT}}$ medulloblastomas can occur at all ages, but are infrequently seen in infants and are more common in children. The $\mathrm{MB}_{\mathrm{GRP} 4}$ subgroup is also more frequent in children, but it can also be seen in both infant and adult medulloblastoma. $\mathrm{MB}_{\mathrm{GRP} 3}$ tumors are frequent in infants and children, but are rare in adults, whereas $\mathrm{MB}_{\mathrm{SHH}}$ subgroups are very frequent in both infants and adults. ${ }^{10,17,19}$ Thus, subgroup analysis using gene expression signatures is providing clinicians with a new tool for improved management of medulloblastoma patients.

In addition to molecular signatures, gene alterations such as mutations in the promotor region of the gene for human telomerase reverse transcriptase (hTERT) have been described in about $20 \%$ of medulloblastomas, and they correlate with a worse prognosis. ${ }^{20}$ Furthermore, the presence of $\mathrm{h} T E R T$ promoter mutations is associated with a higher expression of $\mathrm{h} T E R T$ gene in medulloblastomas, especially in non-infant $\mathrm{MB}_{\mathrm{SHH}}{ }^{20-22}$

The nCounter ${ }^{\circledR}$ system (NanoString Technologies, Seattle, WA, USA) is an automated high-throughput platform designed to evaluate mRNA expression. The mRNA expression levels are directly measured with a high sensitivity in a single multiplexed hybridization reaction. ${ }^{23}$ The nCounter ${ }^{\circledR}$ platform is particularly well-suited for expression analysis of formalin-fixed, paraffin-embedded (FFPE) samples, as the quality of these samples may be highly variable, producing low yields of highly degraded mRNA. ${ }^{24}$ Furthermore, the nCounter ${ }^{\circledR}$ technology does not require further validation, since it does not require enzymatic reactions, it generates reproducible results. ${ }^{23}$ Northcott and colleagues constructed a 22-gene panel that could accurately distinguish medulloblastoma molecular subgroups using the nCounter ${ }^{\circledR}$ platform. The 22 genes they selected have differential expressions associated with each molecular subgroup. ${ }^{25}$

The aim of the present study was to implement the 22-gene panel for molecular classification of medulloblastomas from FFPE blocks using the nCounter ${ }^{\circledR}$ technology in a Brazilian population, and to associate the molecular subgroups with the $\mathrm{h} T E R T$ mutational status and clinicalpathological features of our cohort.

\section{MATERIAL AND METHODS}

\section{Patients}

\section{Brazilian cases}

A series of 104 patients with medulloblastoma were retrieved between 1995 and 2017 from three Brazilian reference centers: the Barretos Cancer Hospital (BCH), Ribeirao Preto Medical School of University of Sao Paulo (FMRP-USP); and Universidade Federal de São Paulo (UNIFESP). In two cases (patients \#87 and \#98; Table S1), we assessed both primary and metastatic tissues. The histologic diagnoses of all cases were reviewed by two expert neuropathologists in accordance with revised 2016 WHO classification, which has histologically defined medulloblastomas as classic, desmoplastic/nodular, extensive nodularity or large cell/anaplastic. ${ }^{26}$ The summary of clinical-pathological and molecular features is presented in the Table S1. This study was approved by the local ethics committee (\#478/2011).

\section{Canadian series}

The molecular data of a series comprising 240 medulloblastomas from the Hospital for Sick Children, University of Toronto, Canada, was kindly provided by Dr. Michael Taylor, and were employed for class prediction. ${ }^{25}$

\section{RNA isolation}

RNA was isolated from three to five $10-\mu$ m-thick sections using RNeasy Mini kit (Qiagen, Venlo, Netherlands) following the manufacturer's instructions. Briefly, following deparaffinization steps, unstained slides were scraped upon a tumor area demarcated on a HE slide. All specimens were submitted to protease digestion followed by nucleic acid isolation followed by DNAse digestion and sample elution. RNA concentrations were assessed by spectrophotometry (NanoDrop; Thermo Fisher Scientific, 
Waltham, MA, USA) and a subset of samples were also evaluated by BioAnalyzer (Agilent Technologies, Inc., Santa Clara, CA, USA).

\section{The nCounter ${ }^{\circledR}$ system (NanoString)}

The medulloblastoma panel was carried out using the NanoString $n$ Counter Elements ${ }^{\mathrm{TM}}$. The custom CodeSet was designed using characteristic genes for each medulloblastoma subgroup as previously employed: $\mathrm{MB}_{\mathrm{SHH}}-P D L I M 3$, EYA1, HHIP, ATOH1, SFRP1; $\mathrm{MB}_{\mathrm{WNT}}$ - WIF1, TNC, GAD1, DKK2, EMX2; $\mathrm{MB}_{\mathrm{GRP} 3}$ - IMPG2, GABRA5, EGFL11, NRL, MAB21L2, NPR3; $\mathrm{MB}_{\mathrm{GRP} 4}-$ KCNA1, EOMES, KHDRBS2, RBM24, UNC5D, OAS1 and three housekeeping genes $(A C T B, G A P D H$ and $L D H A){ }^{25}$ In addition, 11 other cancer genes including hTERT gene were added to the gene panel CodeSet used in this study. The expression analysis of the $\mathrm{h} T E R T$ gene was performed for the present study and the analyses of the other 10 genes will be further reported (manuscript in preparation). All procedures regarding sample preparation, hybridization, detection and scanning were performed according to the manufacturer's instructions (NanoString Technologies, Seattle, WA, USA).

The custom probes (A and B) were designed by IDT (IDT Technologies), containing 35-50 base pairs each and probes were diluted to a final concentration of $0.6 \mathrm{nmol} / \mathrm{L}$ (probe A) and $3.0 \mathrm{nmol} / \mathrm{L}$ (probe $\mathrm{B}$ ) to create the working probe pools. A total amount of $100-300 \mathrm{ng}$ RNA was hybridized with probe pools, hybridization buffer and TagSet reagents and incubated at $67{ }^{\circ} \mathrm{C}$ for $21 \mathrm{~h}$. Then, samples were loaded to the nCounter ${ }^{\circledR}$ PrepStation (NanoString), which automatically performs purification steps and cartridge preparation. Finally, the cartridges containing immobilized reporter complexes were transferred to nCounter $^{\circledR}$ Digital Analyzer (NanoString), a high-resolution setting, which captures up to 280 fields of view (FOVs) per sample providing all gene counts.

\section{DNA isolation and hTERT mutational analysis}

DNA was isolated from three to five sections each $10 \mu \mathrm{m}$ thick using QIAmp DNA Mini kit (QIAgen) as previously reported. ${ }^{20}$ DNA concentrations were assessed by spectrophotometry (NanoDrop).

$\mathrm{h}$ TERT promoter hotspot mutations $(-124 \mathrm{bp} \mathrm{G}>\mathrm{A}$ and -146 bp $\mathrm{G}>\mathrm{A}$ ) were screened by PCR followed by Sanger sequencing as previously described. ${ }^{20,27}$ All samples with mutations were confirmed at least twice.

\section{Data analysis}

Reporter probe counts were captured by nCounter ${ }^{\circledR}$ Digital Analyzer and raw data was collected and pre-processed by nSolver ${ }^{\mathrm{TM}}$ Analysis Software v3.0 (NanoString). For data normalization, NanoStringNorm package was employed (version 18 November, 2015; http://cran.r-project.org/web/ packages/NanoStringNorm). ${ }^{28}$ The normalization method basically consists of four major corrections, which are probelevel background correction, code-count normalization, background correction and sample content normalization by housekeeping genes. The NanoStringNorm package uncovers potential batch-effects, identifies background artifacts and assesses negative/positive controls. Each parameter for all normalizations is associated with plots and all results, diagnostics and expression values were outputted for statistical analyses, heatmaps design and use in class prediction methodologies.

For class prediction analysis of the Brazilian series $(n=104)$, the Canadian series $(n=240)$ was employed to establish a prediction dataset, since all samples had been previously classified for medulloblastoma molecular subgroups. ${ }^{25}$ Raw data from all samples (Brazilian and Canadian series) were normalized and the gene expression values were defined. Normalized data were input for class prediction analysis using the Prediction Analysis for Microarrays (PAM) algorithm. ${ }^{29}$ PAM is a statistical technique for class prediction from gene expression data using nearest shrunken centroids. Class prediction was performed using a machine learning method following these analytical phases: (i) training phase: data from Canadian series were considered for input by pairing with the expected output to train our model employing the PAM algorithm; (ii) validation phase: data from the Brazilian series employing the heatmap clustering as gold-standard were used for initial classification of medulloblastoma molecular subgroups applying the PAM method; (iii) test phase: application of our model to the Brazilian series for class prediction and probability results.

For analysis of hTERT mRNA expression, MannWhitney test was applied for comparing wild-type and $\mathrm{h} T E R T$ mutated cases. Survival analysis was carried out by Kaplan-Meier curves, which were compared by the log-rank test. For disease-specific overall survival (OS), patients who were lost to follow-up were censored considering their last follow-up visit, and patients who died due to external causes or surgical complications were censored considering death date as the last data entry. For all analyses, cancer-specific death was considered as the unfavorable event.

For pre-processing and normalization, statistical analyses, graph constructions, heatmap designs and class 
prediction, the KNIME v.3.2.0 software (KNIME $\mathrm{GmbH}$, Konstanz, Germany) was employed in the $\mathrm{R}$ environment ( $\mathrm{R}$ Foundation, Vienna, Austria). For survival analysis, the IBM SPSS Statistics for Windows version 21.0 (IBM, Armonk, NY, USA) and GraphPad Prism version 6.0 (GraphPad Software, San Diego, CA, USA) were employed. A Cox multivariate analysis considering age and gender as confounders was performed for disease-specific OS analysis. The level of significance for all analyses was $5 \%$.

\section{RESULTS}

\section{Clinicopathological features of Brazilian patients}

We evaluated 104 tumors from patients diagnosed with medulloblastoma (Table 1; Table S1). Thirty-six (35\%) patients were female and $67(65 \%)$ were male (data were unavailable from one patient). The average age at diagnosis was 18 years old (range, 2-56 years), with 11 patients $\leq 4$ years old, 55 cases between 5 and 19 years old, and 37 more than 20 years old (no available information for one patient). Clinical findings showed that $93 \%$ of the patients (92/99) did not have metastasis at diagnosis, whereas $7 \%(7 / 99)$ of the patients presented metastasis at diagnosis (data were not available for five cases). Additionally, $63 \%$ of the patients (59/94) underwent total surgical resection and 37\% (35/94) partial surgical resection. For two patients, surgery was not performed and they were subjected to biopsy only (data were not available for eight cases). Histologic classification showed that 57\% (59/104) had classic histology, 24\% (25/104) desmoplastic/nodular histologic features, 4\% (4/104) anaplastic/large cells, and $12 \%(13 / 104)$ extensive nodularity, and 1\% (1/104) mixed histology. One case was not clearly defined and one case presented inconclusive histological features (Table 1; Table S1).

Overall survival was associated with metastasis at diagnosis $(P=0.04)$ and surgical resection $(P=0.001$; Table S2).

\section{Classification of medulloblastoma molecular subgroups}

We obtained conclusive results from all 106 FFPE samples that comprised 104 primary samples and two metastatic samples. We initially performed an unsupervised clustering to generate heatmaps (Figure S1). The Canadian series provided the known class predictions for comparison to the subgroup findings from the Brazilian cohort. ${ }^{25}$ Following normalization of raw data from both series (Canadian series combined with the Brazilian series; Figure S1), the PAM method was applied for class prediction. Employing a machine learning approach, we successfully classified all medulloblastoma samples according to molecular subgroups (Figs 1,2A). The probability of each subgroup for all samples and the highest probability index for specific molecular subgroups was considered for the final classification. Most samples in each subgroup presented a probability rate higher than $90 \%$. Therefore, the final proportions for classification of the Brazilian medulloblastoma subgroups was $49 \% \mathrm{MB}_{\mathrm{SHH}}(n=51), 18 \% \mathrm{MB}_{\mathrm{WNT}}$ $(n=19), \quad 14 \% \mathrm{MB}_{\mathrm{GRP} 3}(n=15)$ and $18 \% \quad \mathrm{MB}_{\mathrm{GRP} 4}$ $(n=19)$ (Figs 1, 2A; Table 1).

Interestingly, for the two patients (patients \#87 and \#98; Table S1) in which both primary and metastatic tissue could be evaluated, each pair exhibited the same molecular profile $\left(\mathrm{MB}_{\mathrm{SHH}}\right)$.

\section{Association of molecular subgroups with clinicopathological features}

Clinical and molecular features of the molecular subgroup are summarized in Table 1 and described in detail in Table S1. Older age patients were strongly associated wit$\mathrm{h}$ the $\mathrm{MB}_{\mathrm{SHH}}$ subgroup and younger patients with $\mathrm{MB}_{\mathrm{GRP} 3}(P<0.0001$; Table 1$)$. No association by age category $(<4,5-19$ and $>20$ years $)$ and disease outcome was observed $(P=0.1)$. The female gender was strongly associated with $\mathrm{MB}_{\mathrm{WNT}}(P<0.0001$; Table 1$)$.

Table 1 Clinical and molecular characteristics of Brazilian cohort according to the medulloblastoma molecular subgroups

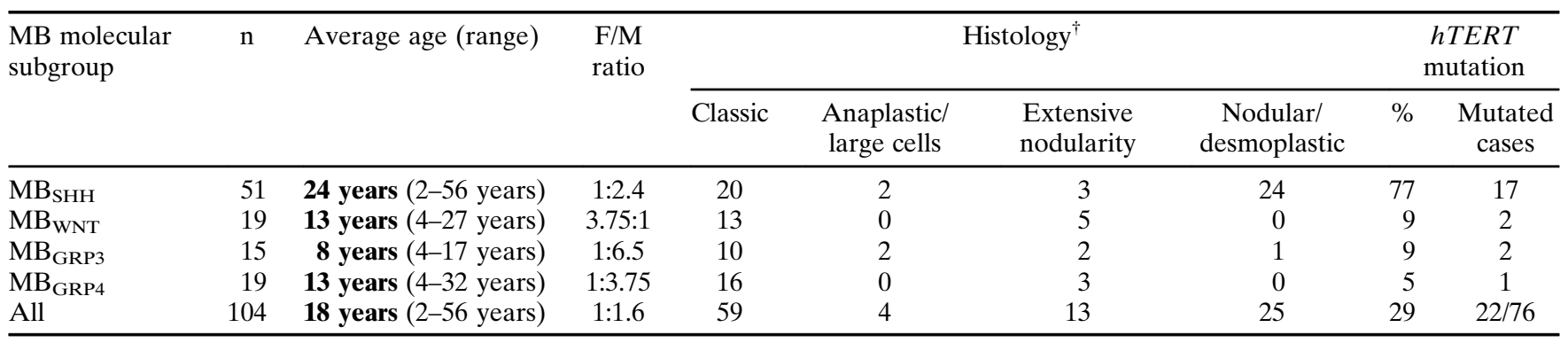

${ }^{\dagger}$ One case from $\mathrm{MB}_{\mathrm{SHH}}$ was classified as Mixed, one case was not possible to classify, and one case no data were available. MB, medulloblastoma; $n$, number of cases; F, female; $\mathrm{M}$, male. 


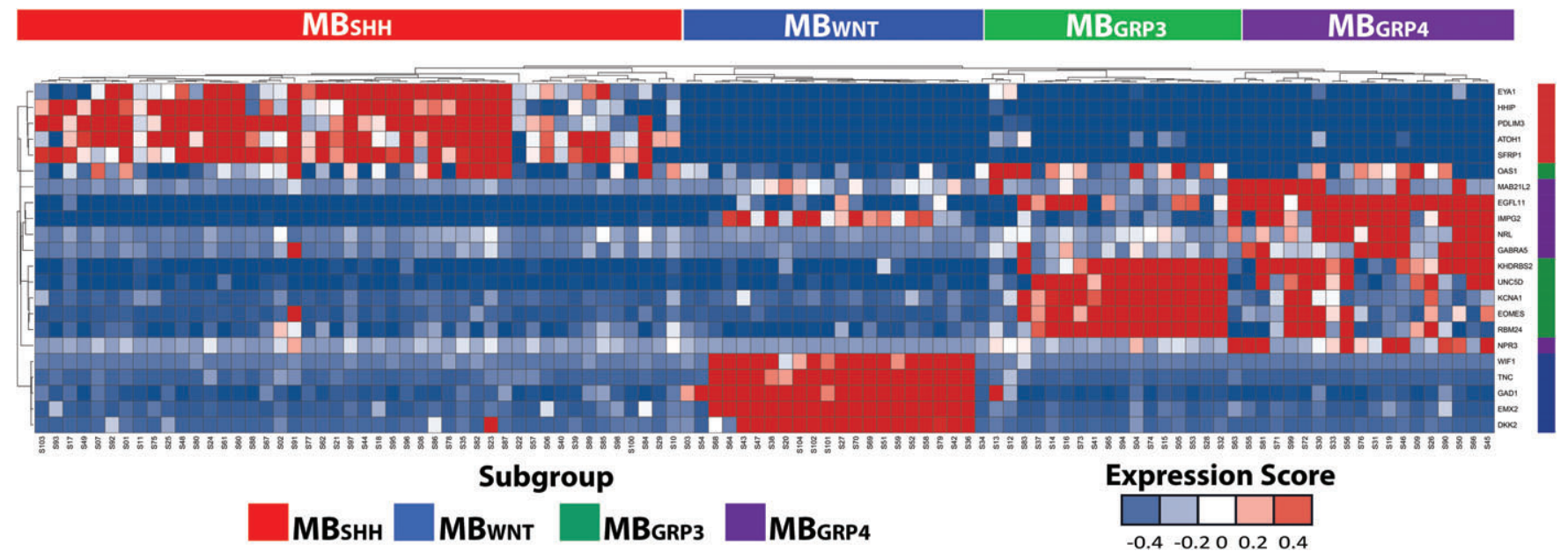

Fig. 1 Heatmap clustering of the Brazilian series $(n=104)$. On the top and on the right side, each colored column represents a medulloblastoma subgroup: $\mathrm{MB}_{\mathrm{SHH}}$, red; $\mathrm{MB}_{\mathrm{WNT}}$, blue; $\mathrm{MB}_{\mathrm{GRP} 4}$, purple; $\mathrm{MB}_{\mathrm{GRP} 3}$, green. Lower right color squares: gene expression scaling from dark blue $(-0.4)$ to dark red $(+0.4)$, with red: increased gene expression; blue: decreased gene expression; white: no differential expression.

Univariate analysis indicated a trend of association between disease-specific OS and medulloblastoma molecular subgroup (log-rank test: $P=0.07$; Fig. 2B; Table S2). The highest 5-year disease-specific OS was observed for the $\mathrm{MB}_{\mathrm{WNT}}(100 \%)$ subgroup, followed by $\mathrm{MB}_{\mathrm{GRP} 3}$ $(73 \%), \mathrm{MB}_{\mathrm{SHH}}(64 \%)$ and $\mathrm{MB}_{\mathrm{GRP} 4}(63 \%)$.

Multivariate analysis showed a higher hazard ratio (HR) for patients who underwent partial surgical resection (HR $=3.73, P=0.006$; Table 2), but no increased HR was observed for age, gender, metastasis at diagnosis, or for molecular subgroup (Table 2).

\section{hTERT mutational status}

Hotspot hTERT promoter mutations ( $-124 \mathrm{bp} \mathrm{G}>\mathrm{A}$ and $-146 \mathrm{bp} \mathrm{G}>\mathrm{A}$ ) were successfully sequenced in $73.1 \%$ (76/104) of tumors. Of these, $29 \%(22 / 76)$ of the cases were mutated (Fig. 3A), with $82 \%$ (18/22) harboring the $-124 \mathrm{bp}$ $\mathrm{G}>\mathrm{A}$ mutation and $18 \%(4 / 22)$ the $-146 \mathrm{bp} \mathrm{G}>\mathrm{A}$ mutation. The frequency of $\mathrm{h} T E R T$ mutations was significantly higher in the $\mathrm{MB}_{\mathrm{SHH}}$ subgroup $(77 \%)$, than in $\mathrm{MB}_{\mathrm{WNT}}$ $(9 \%), \mathrm{MB}_{\mathrm{GRP}}(9 \%)$, and $\mathrm{MB}_{\mathrm{GRP} 4}(5 \%) \quad(\mathrm{p}=0.002$; Table 1; Table S1). The distribution of hTERT mutation was associated with older age as follows: $4.5 \%(1 / 22)$ of mutated cases in patients $\leq 4$ years old, $36.4 \%(8 / 22)$ of mutated cases between 5 and 19 years old, and 59.1\% $(13 / 22)$ of mutated cases in patients more than 20 years old $(P=0.002)$. We extended our analysis to determine whether $\mathrm{h} T E R T$ mutational status correlated with $\mathrm{h} T E R T$ gene expression assessed in the NanoString gene panel, and we found that $\mathrm{h} T E R T$ mutated tumors exhibited a significantly increased hTERT expression $(P=0.03$; Fig. 3B). Since most of the hTERT mutated cases belonged to the (C) 2018 Japanese Society of Neuropathology
$\mathrm{MB}_{\mathrm{SHH}}$ subgroup, we also evaluated whether hTERT mutational status and $\mathrm{h} T E R T$ expression correlated with OS in this specific subgroup, but no association was observed $(P=0.41$ and 0.31 , respectively). Moreover, neither hTERT mutational status nor hTERT expression was associated with $\mathrm{OS}(P=0.34$ and $P=0.72$, respectively; Fig. 3C, D).

\section{DISCUSSION}

Four major medulloblastoma molecular subgroups $\left(\mathrm{MB}_{\mathrm{WNT}}, \mathrm{MB}_{\mathrm{SHH}}, \mathrm{MB}_{\mathrm{GRP} 3}, \mathrm{MB}_{\mathrm{GRP} 4}\right)$ have been described based on specific molecular characteristics, such as mutations and differential expressions of key genes. ${ }^{14-17,30}$ The assignment of medulloblastoma molecular subgroups plays an important role in patient prognostication, ${ }^{30,31}$ and may also be crucial in the design of potential targeted therapies. Herein, we have successfully implemented an effective approach for medulloblastoma molecular subgrouping using FFPE from 104 cases from Brazilian health centers. Additionally, we reported the main clinical and molecular characteristics for each molecular subgroup.

The molecular pathogenesis of medulloblastomas has been partially elucidated by microarray expression profiling and, until recently, this high-throughput technique was the best approach in determining medulloblastoma molecular subgroups. ${ }^{14,15,30}$ However, these earlier platforms required large amounts of high-quality RNA, presented a high variability and are expensive, limiting their application as a routine diagnostic approach. Since there is no current gold-standard method for medulloblastoma molecular subgrouping, the implementation of a robust and inexpensive method is urgently needed. For this reason, 
A Medulloblastoma Molecular Subgroups Distribution

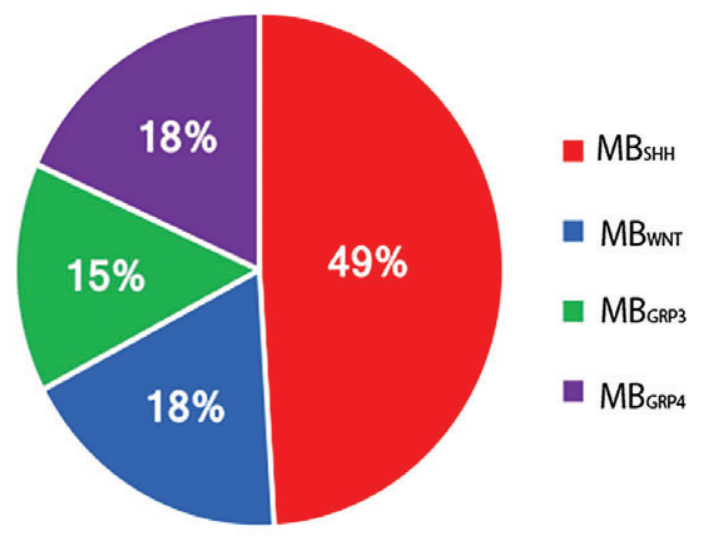

B Disease-specific Overall Survival

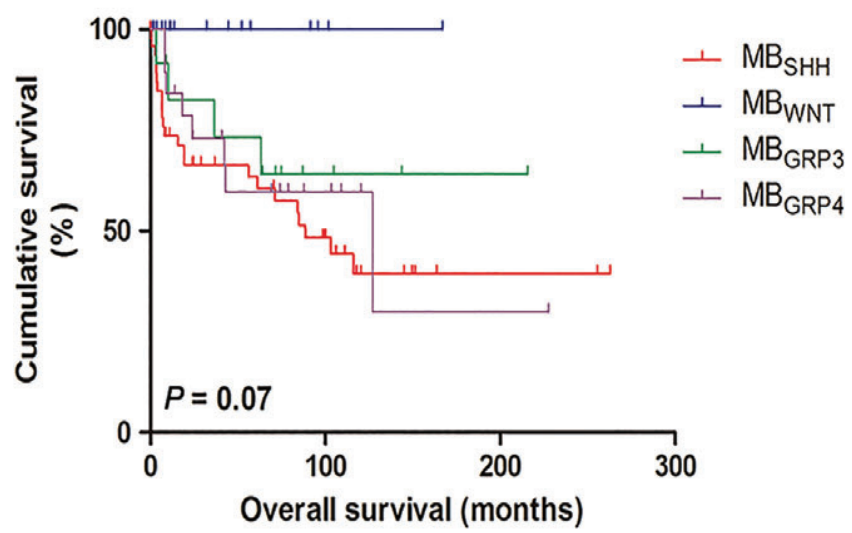

Fig. 2 (A) Medulloblastoma molecular subgroup distributions. Data derived from the Brazilian series $(n=104)$. In this circle chart each colored segment represents a medulloblastoma subgroup: $\mathrm{MB}_{\mathrm{SHH}}$, red; $\mathrm{MB}_{\mathrm{WNT}}$, blue; $\mathrm{MB}_{\mathrm{GRP} 4}$, purple; $\mathrm{MB}_{\mathrm{GRP} 3}$, green. (B) Disease-specific overall survival. Kaplan-Meier curves of overall survival data derived from 99 available patients in the Brazilian series and expressed according to the medulloblastoma molecular subgroups. Cumulative survival as a percentage is shown on the $\mathrm{Y}$-axis and overall survival in months on the $\mathrm{X}$-axis (log-rank test: $P=0.07)$. Live patients, patients who were lost follow up or patients who died due to non-cancer-related causes or surgical complications were censored.

Dr. Taylor's group developed the 22-gene panel for the assignment of medulloblastoma molecular subgroups employing an innovative platform (nCounter ${ }^{\circledR}$ system; NanoString) applied to FFPE samples, which are routinely collected for histopathological analysis. ${ }^{25}$

For actual assignment of the medulloblastoma molecular subgroups based on high-throughput gene expression, it is necessary to employ class prediction analysis. Several algorithms were previously tested by Dr. Taylor's group and the PAM method presented the best scoring index with the lowest misclassification rates compared with other statistical approaches. ${ }^{25}$ This method is faster and can
Table 2 Multivariate proportional hazard analysis on the disease outcome (disease-specific overall survival) of the molecular and clinical characteristics

\begin{tabular}{|c|c|c|c|}
\hline Variables & $n$ & $\begin{array}{l}\text { HR } \\
(95 \% \mathrm{CI})\end{array}$ & $P$-value \\
\hline \multicolumn{4}{|l|}{ Age } \\
\hline Age $<4$ years & 8 & Ref. & Ref. \\
\hline Age group 5-19 years 2 & 40 & $\begin{array}{c}0.53 \\
(0.16-1.76)\end{array}$ & 0.30 \\
\hline Age group $\geq 20$ years 3 & 20 & $\begin{array}{c}0.60 \\
(0.16-2.28)\end{array}$ & 0.45 \\
\hline \multicolumn{4}{|l|}{ Gender } \\
\hline Male & 61 & Ref. & Ref. \\
\hline Female & 20 & $\begin{array}{c}0.97 \\
(0.45-2.12)\end{array}$ & 0.94 \\
\hline \multicolumn{4}{|l|}{ Surgical resection } \\
\hline Total surgical resection & 42 & Ref. & Ref. \\
\hline Partial surgical resection & 26 & $\begin{array}{c}3.73 \\
(1.47-9.47)\end{array}$ & 0.006 \\
\hline \multicolumn{4}{|l|}{ Metastasis at diagnosis } \\
\hline $\begin{array}{l}\text { Absence of metastasis at } \\
\text { diagnosis }\end{array}$ & 63 & Ref. & Ref. \\
\hline $\begin{array}{l}\text { Presence of metastasis at } \\
\text { diagnosis }\end{array}$ & 5 & $\begin{array}{c}0.87 \\
(0.16-4.77)\end{array}$ & 0.88 \\
\hline \multicolumn{4}{|l|}{$\mathrm{h} T E R T$ mutational status } \\
\hline $\mathrm{h} T E R T$ wild-type & 48 & Ref. & Ref. \\
\hline $\mathrm{h} T E R T$ mutated & 20 & $\begin{array}{c}1.76 \\
(0.72-4.32)\end{array}$ & 0.22 \\
\hline \multicolumn{4}{|l|}{ Molecular subgroup } \\
\hline $\mathrm{MB}_{\mathrm{SHH}}$ & 31 & Ref. & Ref. \\
\hline $\mathrm{MB}_{\mathrm{GRP} 3}$ & 11 & $\begin{array}{c}0.91 \\
(0.25-3.26)\end{array}$ & 0.88 \\
\hline $\mathrm{MB}_{\mathrm{GRP} 4}$ & 17 & $\begin{array}{c}0.88 \\
(0.25-3.15)\end{array}$ & 0.84 \\
\hline $\mathrm{MB}_{\mathrm{WNT}}$ & 9 & 0.00() & 0.97 \\
\hline
\end{tabular}

Reference categories: Gender male; molecular subgroup $\mathrm{MB}_{\mathrm{SHH}}$. ${ }^{\dagger} \mathrm{MB}_{\mathrm{WNT}}$ was not possible to estimate because no event (death) was observed for this subgroup (disease-specific overall survival $=100 \%$ ). $P$-values are from Cox proportional hazards analysis. HR, hazard ratio; 95\% CI, 95\% confidence interval; Ref, reference category.

improve accuracy by reducing the effect of intrinsic noise from highly expressed genes, providing easily interpretable results for biologists and clinicians. ${ }^{29}$ For this reason, the PAM method was employed for class prediction in the present study. The gene expression analysis employing the nCounter ${ }^{\circledR}$ technology using the 22-gene panel together with the PAM method for class prediction could classify according to molecular subgroups all medulloblastoma samples analyzed. We also observed the concordance of molecular subgroups in paired primary and metastatic tissues in two patients in accordance with previous data. ${ }^{32}$ In our series, the majority of cases $(49 \%)$ were classified in the $\mathrm{MB}_{\mathrm{SHH}}$ subgroup, followed by $\mathrm{MB}_{\mathrm{WNT}}(18 \%)$, $\mathrm{MB}_{\mathrm{GRP} 4}(18 \%)$ and $\mathrm{MB}_{\mathrm{GRP} 3}(14 \%)$. These frequencies differ slightly from the reported series, that reported higher frequencies of $\mathrm{MB}_{\mathrm{GRP} 3}(20-26 \%)$ and $\mathrm{MB}_{\mathrm{GRP}}$ (35-37\%), which could be explained, at least in part, by the older age (18 years average) of our cohort in comparison with other studies. ${ }^{25,31}$ 
A C TGGGAGGGCCCGGAGGGGGCTGGGCCGGGG ACCCGGG AGGGGTCGGGACGGGG Wild-type

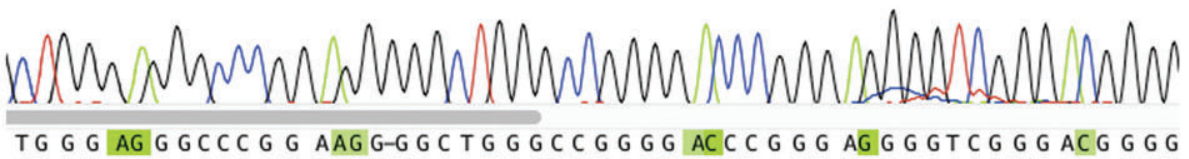
$-124 b p(G>A)$

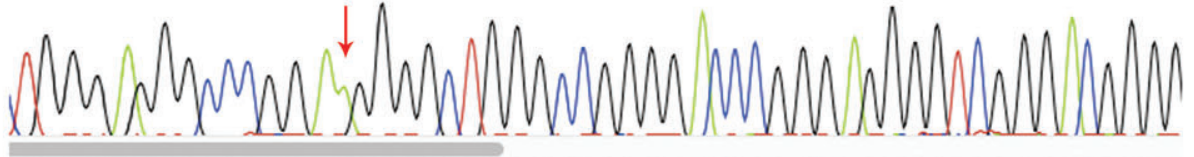

TG G G AG G GCC C G $A G G G G G C T G G G C C G G G G A C C C G G R A G G G G T C G G G$ AC G G G G

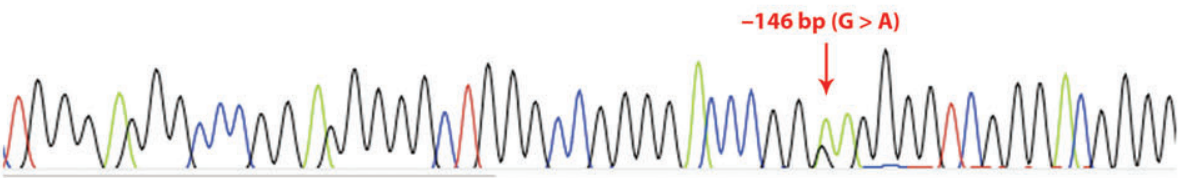

B

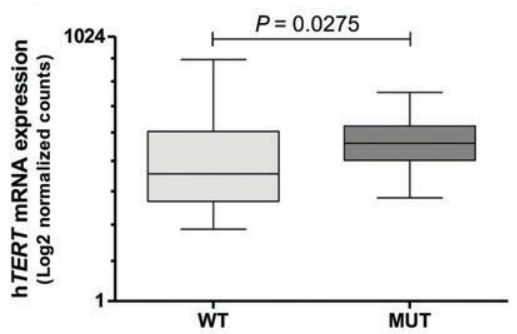

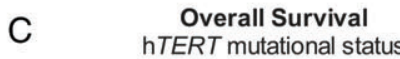

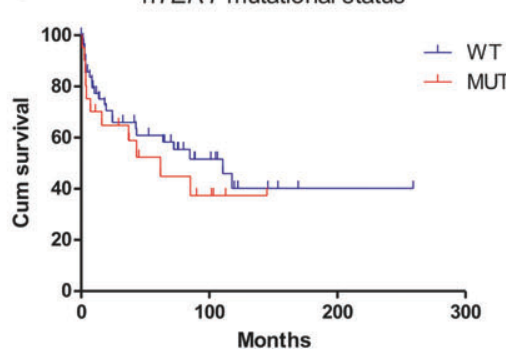

Overall Survival
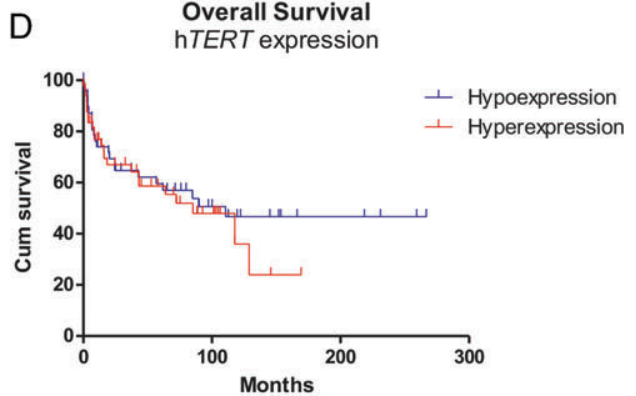

Fig. 3 Analysis of mutation and expression status of hTERT. (A) Nucleotide sequencing of hTERT promotor region showing hotspot promotor mutations ( $-124 \mathrm{bp} \mathrm{G}>\mathrm{A}$ and $-146 \mathrm{G}>\mathrm{A}$ ) identified in medulloblastoma samples. (B) $\mathrm{h} T E R T$ mRNA expression in medulloblastoma carrying hTERT promotor mutations compared with wild-type ones $(n=76$; Mann-Whitney U-test: $P=0.03)$. (C) KaplanMeier curves of overall survival of medulloblastoma patients presenting tumors carrying hTERT promotor mutations (red line) compared with wilt-type (blue line) ones (log-rank test: $P=0.34)$. (D) Kaplan-Meier curves of overall survival of medulloblastoma patients with tumors having either hypoexpression (red line) or overexpression (blue line) of hTERT (log-rank test: $P=0.72$ ).

Hotspot hTERT somatic mutations have been reported in medulloblastomas with frequencies ranging $4-27 \% .{ }^{20-22,33,34}$ In the present study, hTERT mutations were observed in $29 \%$ of the cases, and these mutations were more frequent in $\mathrm{MB}_{\mathrm{SHH}}$ as previously described ${ }^{21,22}$ Moreover, $\mathrm{h} T E R T$ mutations were associated with olderaged patients in accordance with previous studies. ${ }^{20,35}$ Since hTERT-activating promoter mutations are reported to enhance telomerase expression, ${ }^{27}$ we included the $\mathrm{h} T E R T$ gene in the CodeSet of the 22-gene panel for medulloblastoma molecular subgrouping. Unsurprisingly, we observed that $\mathrm{h} T E R T$ mutated cases also exhibited a higher $\mathrm{h} T E R T$ expression.

In the current study, no association was observed between the molecular features (molecular subgroups, $\mathrm{h} T E R T$ mutations status and $\mathrm{h} T E R T$ expression) and OS in the multivariate analysis. Due to the retrospective and multicentric nature of this study, patients were not treated uniformly, which hampers proper outcome analysis.
Our study illustrates the robustness of the NanoString gene expression profiling of FFPE samples, since all 106 cases from different Brazilian institutions provided reliable subgroup classifications. This finding is important because samples from different institutions are likely to have different pre-analytic protocols. Of note, most of the samples in our study had been stored for several years, some of them for more than 20, leading to high levels of nucleic acid degradation, as demonstrated by the difficulties identifying mutational status using traditional analytical methods from the hTERT gene for a subset of cases. These findings suggest that molecular profiling of the 22-gene panel will be important for future medulloblastoma clinical trials utilizing RNA from FFPE samples. Importantly, in the revised 2016 WHO CNS classification, a meaningful integration of genetic/biological information with histopathological features is suggested to enable a more precise medulloblastoma classification. ${ }^{26}$ In this context, the present mRNA-based 22-gene panel accurately 
discriminates the four genetically defined medulloblastoma subgroups, which are $\mathrm{MB}_{\mathrm{WNT}}, \mathrm{MB}_{\mathrm{SHH}}$ and non-MB $\mathrm{MNNT}_{\mathrm{WN}} /$ non-MB $\mathrm{MBH}_{\mathrm{SH}}\left(\mathrm{MB}_{\mathrm{GRP} 3} \text { and } \mathrm{MB}_{\mathrm{GRP} 4}\right)^{26,36,37}$ Yet, in the update of $\mathrm{WHO}$ classification, the $\mathrm{MB}_{\mathrm{SHH}}$ subgroup should further divided in accordance with TP53 status that differentiates two different $\mathrm{MB}_{\mathrm{SHH}}$ disease entities, displaying distinct genetic features, age of diagnosis and outcome. ${ }^{36-38}$

Concluding, we successfully implemented the 22-gene panel originally described by Northcott and colleagues for medulloblastoma molecular classification in a multicenter Brazilian cohort of FFPE cases employing the nCounter ${ }^{\circledR}$ technology. Our findings demonstrate the feasibility of medulloblastoma molecular subgrouping in a routine clinical testing.

\section{ACKNOWLEDGEMENTS}

We thank Barretos Cancer Hospital and FINEP (MCTI/ FINEP/MS/SCTIE/DECIT - BioPlat 1302/13) for partially funding the present study. LFL is supported by Public Ministry of Labor Campinas (Research, Prevention and Education of Occupational Cancer) in Campinas, Brazil. RMR is sponsored by National Council for Scientific and Technological Development (CNPq, Brazil).

\section{DISCLOSURE}

The authors declare they have nothing to disclose.

\section{REFERENCES}

1. McNeil DE, Cote TR, Clegg L, Rorke LB. Incidence and trends in pediatric malignancies medulloblastoma/ primitive neuroectodermal tumor: A SEER update. Surveillance epidemiology and end results. Med Pediatr Oncol 2002; 39: 190-194.

2. Packer RJ, Vezina G. Management of and prognosis with medulloblastoma: Therapy at a crossroads. Arch Neurol 2008; 65: 1419-1424.

3. INCA. Instituto Nacional do Câncer - Estimativa 2010: Incidência de câncer no Brasil. 2009 [cited 12 April 2018]. Available from URL: http://www2. inca.gov.br/wps/wcm/connect/agencianoticias/site/ home/noticias/2009/lancamento_estimativa_2010.

4. Chan AW, Tarbell NJ, Black PM et al. Adult medulloblastoma: Prognostic factors and patterns of relapse. Neurosurgery 2000; 47: 623-631; discussion 31-2.

5. Ellison DW. Childhood medulloblastoma: Novel approaches to the classification of a heterogeneous disease. Acta Neuropathol 2010; 120: 305-316.

6. Taylor RE, Bailey CC, Robinson KJ et al. Outcome for patients with metastatic (M2-3) medulloblastoma treated with SIOP/UKCCSG PNET-3 chemotherapy. Eur J Cancer 2005; 41: 727-734.

7. Gajjar A, Hernan R, Kocak M et al. Clinical, histopathologic, and molecular markers of prognosis: Toward a new disease risk stratification system for medulloblastoma. J Clin Oncol 2004; 22: 984-993.

8. Gilbertson RJ, Ellison DW. The origins of medulloblastoma subtypes. Annu Rev Pathol 2008; 3: 341-365.

9. Gilbertson R, Wickramasinghe C, Hernan R et al. Clinical and molecular stratification of disease risk in medulloblastoma. Br J Cancer 2001; 85: 705-712.

10. Kim W, Choy W, Dye J et al. The tumor biology and molecular characteristics of medulloblastoma identifying prognostic factors associated with survival outcomes and prognosis. J Clin Neurosci 2011; 18: 886-890.

11. de Haas T, Hasselt N, Troost D et al. Molecular risk stratification of medulloblastoma patients based on immunohistochemical analysis of MYC, LDHB, and CCNB1 expression. Clin Cancer Res 2008; 14: 4154-4160.

12. von Bueren AO, Kortmann $\mathrm{RD}$, von Hoff $\mathrm{K}$ et al. Treatment of children and adolescents with metastatic medulloblastoma and prognostic relevance of clinical and biologic parameters. J Clin Oncol 2016; 34: 4151-4160.

13. Ramaswamy V, Remke M, Bouffet E et al. Risk stratification of childhood medulloblastoma in the molecular era: The current consensus. Acta Neuropathol 2016; 131: 821-831.

14. Thompson MC, Fuller C, Hogg TL et al. Genomics identifies medulloblastoma subgroups that are enriched for specific genetic alterations. J Clin Oncol 2006; 24: 1924-1931.

15. Kool M, Koster J, Bunt $\mathrm{J}$ et al. Integrated genomics identifies five medulloblastoma subtypes with distinct genetic profiles, pathway signatures and clinicopathological features. PLoS One 2008; 3: e3088.

16. Northcott PA, Korshunov A, Witt $\mathrm{H}$ et al. Medulloblastoma comprises four distinct molecular variants. $J$ Clin Oncol 2011; 29: 1408-1414.

17. Taylor MD, Northcott PA, Korshunov A et al. Molecular subgroups of medulloblastoma: The current consensus. Acta Neuropathol 2012; 123: 465-472.

18. Kool M, Korshunov A, Remke M et al. Molecular subgroups of medulloblastoma: An international metaanalysis of transcriptome, genetic aberrations, and clinical data of WNT, SHH, Group 3, and Group 4 medulloblastomas. Acta Neuropathol 2012; 123: 473-484.

19. Remke M, Hielscher T, Northcott PA et al. Adult medulloblastoma comprises three major molecular variants. J Clin Oncol 2011; 29: 2717-2723.

20. Viana-Pereira M, Almeida GC, Stavale JN et al. Study of hTERT and histone 3 mutations in medulloblastoma. Pathobiology 2017; 84: 108-113. 
21. Remke M, Ramaswamy V, Peacock J et al. TERT promoter mutations are highly recurrent in SHH subgroup medulloblastoma. Acta Neuropathol 2013; 126: 917-929.

22. Lindsey JC, Schwalbe EC, Potluri S, Bailey S, Williamson D, Clifford SC. TERT promoter mutation and aberrant hypermethylation are associated with elevated expression in medulloblastoma and characterise the majority of non-infant SHH subgroup tumours. Acta Neuropathol 2014; 127: 307-309.

23. Geiss GK, Bumgarner RE, Birditt B et al. Direct multiplexed measurement of gene expression with colorcoded probe pairs. Nat Biotechnol 2008; 26: 317-325.

24. Veldman-Jones MH, Brant R, Rooney C et al. Evaluating robustness and sensitivity of the NanoString technologies nCounter platform to enable multiplexed gene expression analysis of clinical samples. Cancer Res 2015; 75: 2587-2593.

25. Northcott PA, Shih DJ, Remke M et al. Rapid, reliable, and reproducible molecular sub-grouping of clinical medulloblastoma samples. Acta Neuropathol 2012; 123: 615-626.

26. Louis DN, Perry A, Reifenberger G et al. The 2016 World Health Organization classification of tumors of the central nervous system: A summary. Acta Neuropathol 2016; 131: 803-820.

27. Batista R, Cruvinel-Carloni A, Vinagre $\mathrm{J}$ et al. The prognostic impact of TERT promoter mutations in glioblastomas is modified by the rs2853669 single nucleotide polymorphism. Int $J$ Cancer 2016; 139: 414-423.

28. Waggott D, Chu K, Yin S, Wouters BG, Liu FF, Boutros PC. NanoStringNorm: An extensible R package for the pre-processing of NanoString mRNA and miRNA data. Bioinformatics 2012; 28: 1546-1548.

29. Tibshirani R, Hastie T, Narasimhan B, Chu G. Diagnosis of multiple cancer types by shrunken centroids of gene expression. Proc Natl Acad Sci U S A 2002; 99: 6567-6572.

30. Cho YJ, Tsherniak A, Tamayo $\mathrm{P}$ et al. Integrative genomic analysis of medulloblastoma identifies a molecular subgroup that drives poor clinical outcome. J Clin Oncol 2011; 29: 1424-1430.

31. Thompson EM, Hielscher T, Bouffet E et al. Prognostic value of medulloblastoma extent of resection after accounting for molecular subgroup: A retrospective integrated clinical and molecular analysis. Lancet Oncol 2016; 17: 484-495.
32. Wang X, Dubuc AM, Ramaswamy V et al. Medulloblastoma subgroups remain stable across primary and metastatic compartments. Acta Neuropathol 2015; 129: 449-457.

33. Killela PJ, Reitman ZJ, Jiao Y et al. TERT promoter mutations occur frequently in gliomas and a subset of tumors derived from cells with low rates of self-renewal. Proc Natl Acad Sci U S A 2013; 110: 6021-6026.

34. Schwalbe EC, Lindsey JC, Nakjang $\mathrm{S}$ et al. Novel molecular subgroups for clinical classification and outcome prediction in childhood medulloblastoma: A cohort study. Lancet Oncol 2017; 18: 958-971.

35. Koelsche C, Sahm F, Capper D et al. Distribution of TERT promoter mutations in pediatric and adult tumors of the nervous system. Acta Neuropathol 2013; 126: $907-915$.

36. Pietsch T, Haberler C. Update on the integrated histopathological and genetic classification of medulloblastoma - A practical diagnostic guideline. Clin Neuropathol 2016; 35: 344-352.

37. Louis D, Ohgaki H, Wiestler O, Cavenee W. WHO Classification of Tumours of the Central Nervous System, 4th edn. Lyon, France: International Agency for Research on Cancer, 2016. 408 p.

38. Zhukova N, Ramaswamy V, Remke $M$ et al. Subgroup-specific prognostic implications of TP53 mutation in medulloblastoma. J Clin Oncol 2013; 31: 2927-2935.

\section{SUPPORTING INFORMATION}

Additional supporting information may be found in the online version of this article at the publisher's website: http://onlinelibrary.wiley.com/doi//suppinfo.

Table S1 Clinical, pathological and molecular characteristics and outcomes of the Brazilian series of medulloblastomas analyzed in the present study.

Table S2 Univariate analysis of disease-specific overall survival associated with clinical, pathological and molecular characteristics of the Brazilian series of medulloblastomas.

Figure S1 Heatmap clustering of Canadian series $(n=240)$ class prediction control dataset combined with the Brazilian cohort series $(n=104)$. On the top and on the right side, each colored column represents a medulloblastoma subgroup: $\mathrm{MB}_{\mathrm{SHH}}$, red; $\mathrm{MB}_{\mathrm{WNT}}$, blue; $\mathrm{MB}_{\mathrm{GRP} 4}$, purple; $\mathrm{MB}_{\mathrm{GRP} 3}$, green. Lower right square: gene expression scaling from dark red to dark blue; red, increased gene expression; blue, decreased gene expression (as described in legend to Fig. 1). 